\title{
Evaluación estética de seis tipos de coronas para dientes primarios
}

Héctor Alejandro Ramírez Peña ${ }^{1}$,

Eyra Eloyra Rangel Padilla ${ }^{1}$, Héctor R. Martínez-Menchaca²,

Gerardo Rivera Silva ${ }^{3}$,

Gabriela Arredondo Campo ${ }^{4}$,

Natalia Barba Borrego $0^{4}$,

Gustavo Israel Martínez,

Roberto Valencia Hitte ${ }^{5}$

\section{Resumen}

Objetivo: Evaluar las preferencias estéticas en relación con el color y la forma de coronas primarias utilizadas para dientes incisivos superiores primarios, mediante la realización de una encuesta a miembros de la Academia Mexicana de Odontología Pediatrica (AMOP). Material y Métodos: Se establecieron seis grupos de estudio con seis coronas diferentes: grupo 1, coronas de zirconia EZ-Pedo; grupo 2, coronas de zirconia NuSmile Zr; grupo 3 , coronas estéticas hechas en el consultorio; grupo 4, coronas de fundas de celuloide; grupo 5, coronas estéticas prefabricadas NuSmile signature; y grupo 6, coronas estéticas fenestradas. Se llevaron a cabo encuestas con la finalidad de conocer las preferencias estéticas de estas diferentes coronas, con la finalidad de conocer cuál es la mejor opción para su uso en el consultorio dental. Resultados: Noventa miembros de la AMOP realizaron una encuesta válida, y se determinó que el grupo 4 fue el mejor evaluado, seguido de los grupos 2, 5, 1, 6 y 3. Se identificaron diferencias significativas entre los diferentes grupos. Conclusiones: Las coronas de fundas de celuloide fueron seleccionadas como mejor alternativa de uso en los dientes primarios anteriores, por parte de los miembros de la AMOP; asimismo, se consideró a las coronas de zirconia como una buena opción terapéutica. Es recomendable que se implemente el tratamiento estético en dientes primarios, para realizar un tratamiento integral.

Palabras Clave: Deciduos, Zirconio, coronas con frente estético, odontología pediátrica

\footnotetext{
1. Profesor en Odontología Pediatrica en la Universidad Autónoma de Nuevo León UANL, Monterrey, NL, MEX.

2. Vice-Chair of Department of Orthodontics, Pediatric Dentistry and Special Care at University of Louisville School of Dentistry, Louisville, KY, USA.

3. Laboratorio de Ingeniería Tisular y Medicina Regenerativa, Departamento de Ciencias Básicas, Universidad de Monterrey. NL, MEX.

4. Residente de la Maestría en Ciencias en el área de Odontopediatria en la Univer-sidad Autónoma de Nuevo León UANL, Monterrey, NL, MEX.

5. Profesor en Odontología Pediatrica en la Universidad Tecnológica de México UNITEC, Ciudad de México, MEX.
} 


\section{Avaliação estética de seis tipos de coroas para dentes decíduos}

\section{Resumo}

Objectivo: Avaliar as preferências estéticas sobre cor e forma das coroas primários utilizados para a incisivos superiores primários, através da realização de uma pesquisa com membros da Academia Mexicana de Odontopediatria (Amop). Material e Métodos: Seis grupos de estudo com seis coroas diferentes foram estabelecidas: Grupo 1, coroas de zircônia EZ-Pedo; Grupo 2, coroas de zircônia NuSmile Zr; Grupo 3, coroas estéticas feitas no escritório; Grupo 4 casos coroas celulóide; Grupo 5, coroas estéticas prefabricados assinatura NuSmile; e Grupo 6, fenestrado coroas estéticas. Eles realizaram pesquisas, a fim de satisfazer as preferências estéticas destas coroas estéticas diferentes, a fim de saber qual é a melhor escolha para uso no consultório odontológico. Resultados: Noventa membros AMOP fez um levantamento válido, e determinou-se que o Grupo 4 foi o melhor avaliado, seguido pelos grupos foram identificados 2, 5, 1, 6 e 3 . As diferenças significativas entre os grupos. Conclusões. As coroas de tampas de celulóide foram selecionados como o melhor uso alternativo nos dentes primários acima, por membros da Amop; Também as coroas de zircônia foi considerado como uma boa opção terapêutica. Recomenda-se que o tratamento cosmético é implementado em dentes primários, para um tratamento abrangente.

Palavras-chave: Decíduos, Zircônio, facetas dentarias, Odontopediatra.

Original article

\section{Aesthetic evaluation of six types of crowns for primary teeth}

\section{Abstract}

Objective: Evaluate the aesthetic preferences regarding color and shape of primary crowns used for primary upper incisors, by conducting a survey of members of the Mexican Academy of Pediatric Dentistry (AMOP). Material and Methods: Six study groups with six different crowns were established: Group 1, zirconia crowns EZ-PEDO; Group 2, zirconia crowns NuSmile Zr; Group 3 aesthetic crowns made in the office; Group 4 cases strip crowns; Group 5, aesthetic crowns prefabricated NuSmile signature; and Group 6, fenestrated esthetic crowns. They conducted surveys in order to meet the esthetic preferences of six different aesthetic crowns, in order to know the best choice for use in the dental office. Results: Ninety AMOP members made a valid survey, and it was determined that Group 4 was the best evaluated, followed by the groups 2, 5, 1, 6 and 3. Significant differences between groups were identified. Conclusions: Members of the AMOP selected strip crowns as the best alternative use in the above primary teeth; furthermore zirconium crowns were considered as a good alternative. It is recommended that the cosmetic treatment should be implemented in primary teeth, for a comprehensive treatment.

Keywords: Primary teeth, zirconia, veneers esthetic crowns, pediatric dentistry. 


\section{Introducción}

La restauración de los dientes incisivos superiores, que se pueden ver severamente dañados durante la etapa de dentición primaria, es un reto clínico y estético. Las lesiones por caries en las piezas anteriores hasta hace algunos años se resolvían con extracciones o bien con la cobertura mediante el uso de coronas de acero-cromo. ${ }^{1}$ Actualmente, la caries dental en niños es un problema de salud pública serio, ya que según datos de la Academia Americana de Odontología Pediátrica (AAPD por sus siglas en inglés), se hace mención de la pérdida de uno o más dientes cariados $\mathrm{u}$ obturados antes de los setenta y uno meses de edad. ${ }^{2}$ Los motivos más frecuentes de la pérdida dentaria en la niñez son la caries de la infancia temprana y el traumatismo dental. Durante la última década, se ha despertado una perspectiva estética por parte de los padres de familia en relación con la dentición primaria de sus hijos, condicionado el que se busquen y generen nuevas alternativas de tratamiento. ${ }^{3}$

Las restauraciones en la dentición primaria deberían restablecer la funcionalidad, ser durables y mantener una estética adecuada; por este motivo, en las situaciones en donde no se obtengan estas características al cien por ciento, es imperativo buscar las mejores opciones. En la odontopediatría contemporánea se cuenta con diferentes alternativas estéticas, que van desde restauraciones intradentales (resinas compuestas, ionómeros de vidrio, compómeros), hasta restauraciones extradentales (coronas de acero, coronas de fundas de celuloide, coronas fenestradas, coronas de metal con frente estético realizadas en el consultorio o las que son prefabricadas). ${ }^{4-5}$ Las coronas disponibles para la restauración de los incisivos primarios incluyen a las que el material se adhiere directamente sobre el diente (strip crowns), que generalmente es resina compuesta, y las que son cementadas sobre el diente (coronas de acero cromo). ${ }^{6}$

Varias alternativas terapéuticas se han utilizado para restaurar la zona anterosuperior de los dientes primarios con la finalidad de dar una apariencia estética, y tener una opción diferente a la corona metálica. Una de las primeras propuestas diferentes al uso de las coronas metálicas, la hace Kopel (1967) con las coronas fenestradas (facial cut-out SSC), consistía en una fenestración de la cara vestibular de la corona para ser rellenadas con resina compuesta. ${ }^{7}$ Por otra parte, las coronas de celuloide (strip crown, pedoform), fueron introducidas en los setenta, contienen a manera de fundas de celuloide a la resina compuesta para ser adheridas posteriormente al diente preparado. ${ }^{8}$ Las coronas prefabricadas con frente estético (resin-veneered SSC), introducidas en los noventa, son coronas prefabricadas con materiales como resinas compuestas o bien termoplásticas, están adheridas a coronas de acero-cromo (NuSmile crowns, Kinder-Crowns, Cheng-Crowns, Dura Crowns, Whiter Biter II). ${ }^{9-11}$ Las coronas metálicas con frente estético previamente hechas en el consultorio (chair side), así como las coronas de policarbonato (Artglass), han surgido en su momento como alternativas terapéuticas por ser materiales excelentes para el recubrimiento de dientes primarios; sin embargo, algunas han fallado en durabilidad, fracturas, desalojo, color, etc., por lo que se continua en la búsqueda de aquellas que proporcionen el mejor resultado.

Las nuevas coronas hechas de zirconia monolítica, introducidas al mercado a finales de la década pasada, ofrecen una alternativa estética adecuada y tienen como principal ventaja el color y la durabilidad; empero, los reportes de su uso 


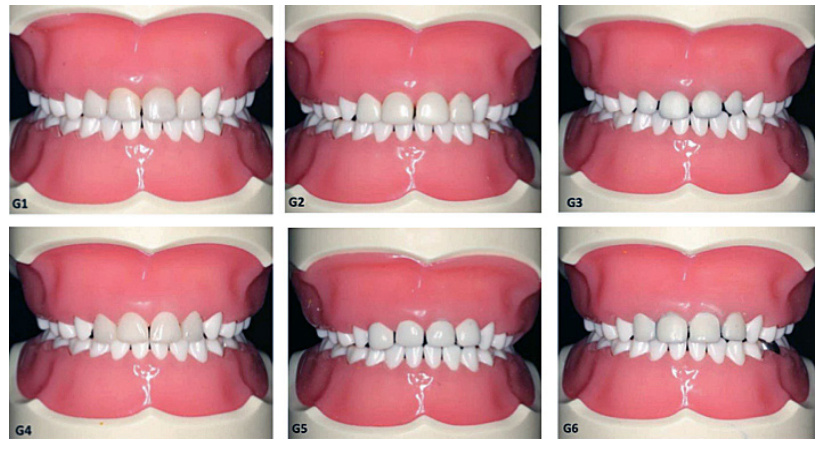

Figura 1. Imágenes fotográficas de las coronas utilizadas en los cuatro incisivos superiores de los diferentes tipodontos. G1. Grupo 1, coronas de zirconia monolítica EZ Pedo®. G2. Grupo 2, coronas de zirconia monolítica NuSmile Zr®. G3. Grupo 3, coronas de acero-cromo estéticas hechas en el consultorio. G4. Grupo 4, coronas de funda de celuloide 3M®. G5. Grupo 5, coronas de metal con frente estético prefabricadas Nusmile Signature®. G6. Grupo 6, coronas de acero cromo fenestradas.

en dientes primarios se han limitado solamente a reportes de casos aislados. Son consideradas como la mejor alternativa estética de todas, aunque tiene sus indicaciones precisas. ${ }^{12,13}$

El objetivo del estudio es conocer las preferen-

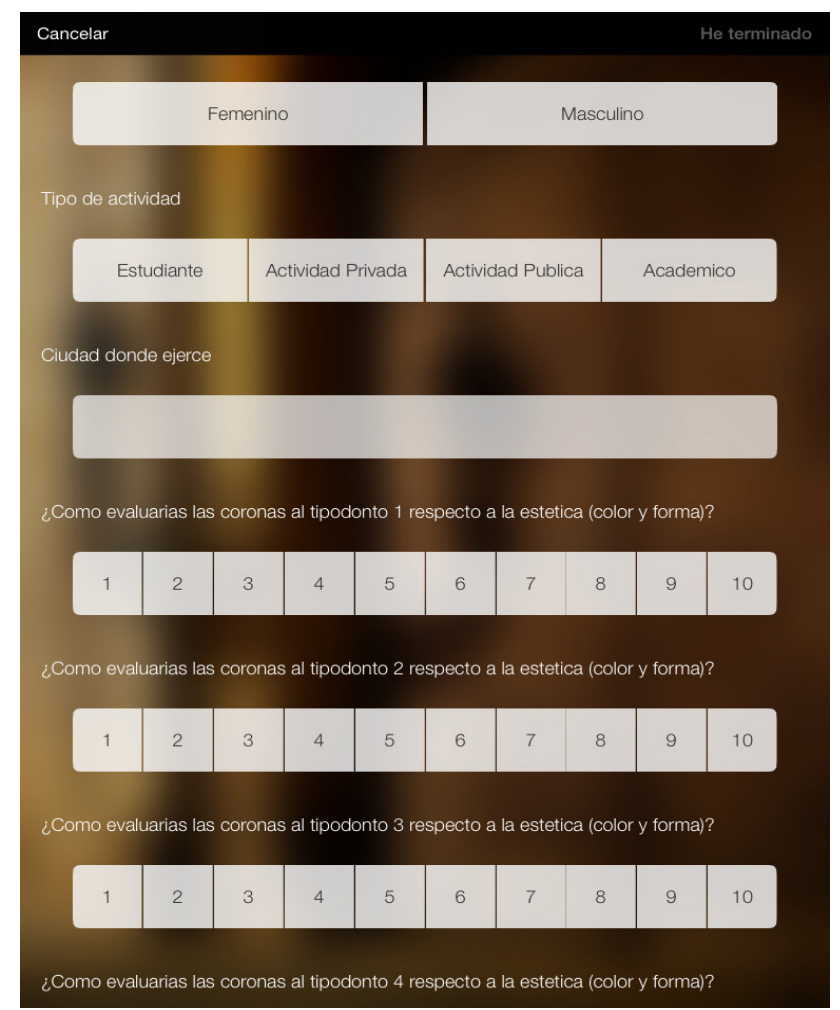

cias estéticas por parte de miembros de la Academica Mexicana de Odontologia Pediatrica (AMOP), en relación con la forma y el color de las coronas primarias estéticas para dientes incisivos anteriores superiores primarios.

\section{Materiales y Métodos}

Fueron mostrados seis modelos de estudio de dentición primaria utilizando un modelo de encía blanda $\left(\mathrm{Nissin}{ }^{\circledR}\right)$; a los cuales se realizaron 6 diferentes tipos de tratamiento de restauraciones estéticas en los cuatro dientes anteriores superiores, en cada uno de los tipodontos (figura 1). Por lo cual se establecieron seis grupos de estudio: grupo 1 (ZEP) donde se utilizaron coronas de zirconia monolítica (EZ PEDO ${ }^{\circledR}$, Loomis, Calif. USA); grupo 2 (ZNS), coronas de zirconia monolítica (NuSmile ZR ${ }^{\circledR}$, Pediatric Crowns, Houston Tex. USA); grupo 3 (MEHC), coronas de acero-cromo $\left(3 \mathrm{M}^{\circledR}\right.$, St Paul Minn), hechas en el laboratorio del

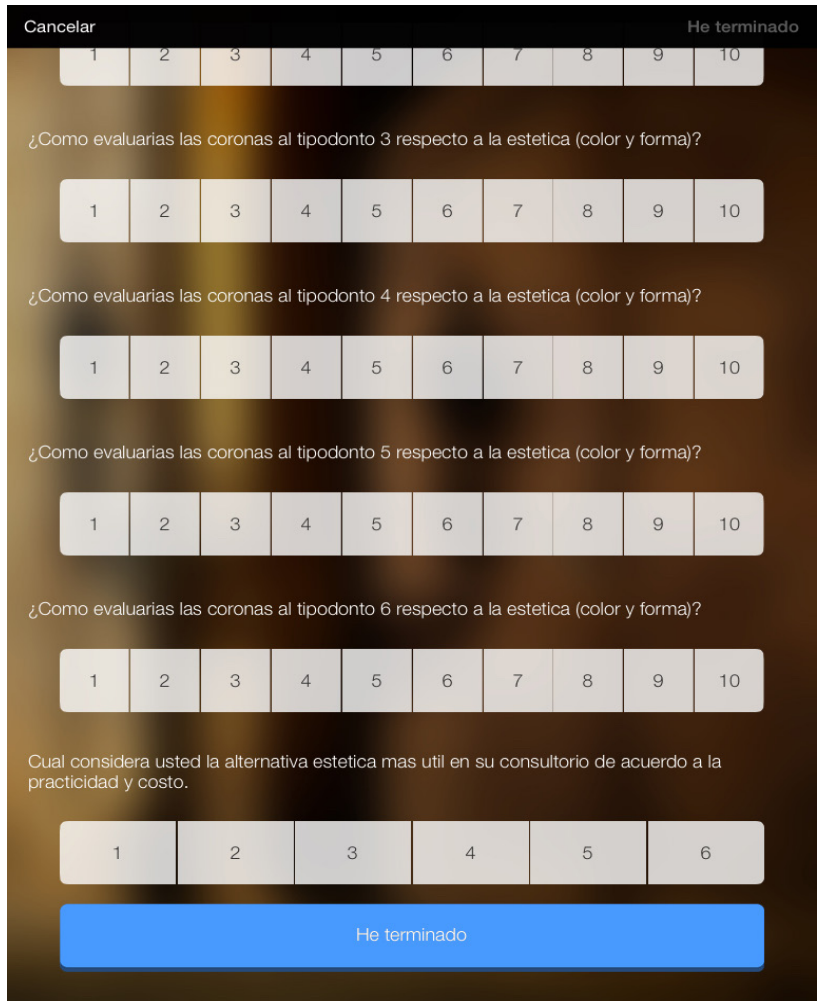

Figura 2. Imágenes de la encuesta realizada por la aplicación Loop Survey. 


\begin{tabular}{|c|c|c|c|c|c|c|}
\hline & Tipodonto 1 & Tipodonto 2 & Tipodonto 3 & Tipodonto 4 & Tipodonto 5 & Tipodonto 6 \\
\hline Media & 6.41 & 7.66 & 4.63 & 8.80 & 6.91 & 5.06 \\
\hline Mediana & 7 & 8 & 5 & 9 & 7 & 5 \\
\hline Moda & 7 & 9 & 5 & 9 & 7 & 7 \\
\hline $\begin{array}{l}\text { Desviación } \\
\text { estándar }\end{array}$ & 1.68 & 1.63 & 1.95 & 1.14 & 1.73 & 1.95 \\
\hline Varianza & 2.83 & 2.66 & 3.79 & 1.29 & 2.98 & 3.81 \\
\hline Mínimo & 1 & 3 & 1 & 4 & 2 & 1 \\
\hline Máximo & 9 & 10 & 9 & 10 & 10 & 9 \\
\hline Rango & 8 & 7 & 8 & 6 & 8 & 8 \\
\hline \multirow{2}{*}{ IC: $95 \%$} & 6.76 & 8.00 & 5.04 & 9.04 & 7.27 & 5.46 \\
\hline & 6.06 & 7.31 & 4.23 & 8.55 & 6.55 & 4.65 \\
\hline
\end{tabular}

Tabla 1. Estadística descriptiva de la evaluación estética (color y forma) por tipodonto.

consultorio con frente de resina compuesta en la cara vestibular; la técnica consiste en realizar un arenado de la superficie vestibular, con aplicación del acondicionamiento del metal, utilización del adhesivo universal $3 \mathrm{M}^{\circledR}$, y posteriormente se coloca el opacador de metal (agente enmascarante) y resina, se fotopolimeriza y se lleva a un horno de luz y calor para que se efectúe polimerización lenta. Grupo 4 (CSC), coronas de funda de celuloide (Pedoform Strip crowns forms, 3M${ }^{\circledR}$ St Paul Minn) obturándose con resina $\mathrm{Z} 350^{\circledR}$ sombra A1; grupo 5 (MEP), coronas de metal con frente estético prefabricadas (NuSmile Signature ${ }^{\circledR}$, Pediatric Crowns, Houston Tex. USA); y el grupo 6 (MEF), coronas de acero cromo fenestradas $\left(3 \mathrm{M}^{\circledR}\right.$, St Paul Minn), en donde a las coronas previamente cementadas, se les hace una ventana en la cara vestibular, posteriormente se aplica opacador y se obtura con resina compuesta Z-350 3M ${ }^{\circledR}$ Sombra A1.

Se aplicó una encuesta directa a miembros que asistieron al curso Intermedio de la Academia Mexicana de Odontología Pediatrica (AMOP), utilizando un app LOOP SURVEY ${ }^{\circledR}$ (figura 2), quienes accedieron compartir su experiencia y calificar a los seis grupos establecidos. En las primeras seis preguntas se estableció un rango entre 0 y 10 puntos, para obtener la calificación. La única condición que se les pidió, fue el de no observar las coronas en su cara palatina, para que no identificaran la marca de la corona utilizada. Los encuestados que observaron la cara palatina, fueron descartados. La recopilación de la información fue realizada y concentra- 


\begin{tabular}{|c|c|c|c|c|c|c|c|c|}
\hline \multirow[b]{2}{*}{ Tipodonto } & \multicolumn{2}{|c|}{ Académico } & \multicolumn{2}{|c|}{$\begin{array}{c}\text { Actividad } \\
\text { privada }\end{array}$} & \multicolumn{2}{|c|}{ Estudiante } & \multicolumn{2}{|c|}{ Total } \\
\hline & $\mathbf{n}$ & $\%$ & $\mathbf{n}$ & $\%$ & $\mathbf{n}$ & $\%$ & $\mathbf{n}$ & $\%$ \\
\hline 1 & 0 & 0.00 & 1 & 4.55 & 0 & 0.00 & 1 & 1.11 \\
\hline 2 & 8 & 22.86 & 5 & 22.73 & 1 & 3.03 & 14 & 15.56 \\
\hline 4 & 25 & 71.43 & 13 & 59.09 & 31 & 93.94 & 69 & 76.67 \\
\hline 5 & 1 & 2.86 & 2 & 9.09 & 0 & 0.00 & 3 & 3.33 \\
\hline 6 & 1 & 2.86 & 1 & 4.55 & 1 & 3.03 & 3 & 3.33 \\
\hline Total & 35 & 100 & 22 & 100 & 33 & 100 & 90 & 100 \\
\hline
\end{tabular}

Tabla 2. Alternativa estética más útil en su consultorio de acuerdo a la practicidad y costo según el tipo de actividad. $X^{2}=14.08 . p=0.0796$

da por el entrevistado en una tableta (Ipad $2^{\circledR}$, Apple Inc, Cupert Calif. USA), lo que permitió que la aplicación fuera de manera rápida y confidencial.

\section{Resultados}

La encuesta fue realizada a 170 miembros de la AMOP, de los cuales fueron excluidos 80 encuestados, debido a que no reunieron todos los criterios de inclusión. De los 90 miembros que realizaron la evaluación con éxito; 39\% se dedicaba a una actividad académica y privada $(\mathrm{n}=35)$, el 25\% únicamente a una actividad privada $(n=23)$ y finalmente el $36 \%$ fueron estudiantes de posgrado $(n=32)$.

En la tabla 1, se muestra que el grupo de coronas de fundas de celuloide pertenecientes al grupo 4 (CSC: Coronas Strip Crowns), obtuvo la mejor calificación con respecto a los otros cinco grupos (8.8); el grupo 2, con coronas de zirconia NuS- mile ZR (ZNS) fue el segundo mejor evaluado; mientras que el tercer grupo mejor evaluado fue el grupo 5 (MEP: Metálicas estéticas prefabricadas); el cuarto grupo mejor evaluado fue el grupo 1, con coronas de zirconia EZ-Pedo (ZEP); el quinto mejor evaluado fueron las coronas metálicas fenestradas (MEF) grupo 6; y finalmente la coronas metálicas con frente estético hechas en el consultorio que se correspondían al grupo 3 (MEHC), fueron las que obtuvieron la calificación más baja (Tabla 2).

Los diferentes grupos mostraron diferencias significativas ( $p>0.05$ ) entre ellos, en la selección de las coronas estéticas primarias más adecuadas (Tabla 3); asimismo, en la tabla 4 se muestra que el grupo 4 (NSC) se diferencia de los 5 grupos restantes ( $p>0.05)$. El grupo 1 y el grupo 2 no mostraron diferencias significativas ( $\mathrm{p}=.298$ y $\mathrm{p}=0.40$, respectivamente), comparados con el grupo 5 (MES); mientras que el grupo 3 (MEHC) no mostró diferencias significativas $(\mathrm{P}=.555)$ con el grupo 6 (MEF). 


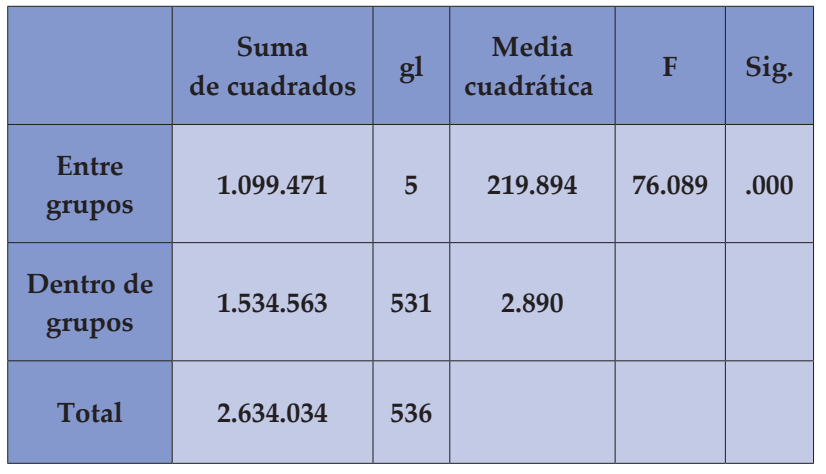

Tabla 3. Análisis de varianza de las evaluaciones según el tipodonto elegido de acuerdo a la practicidad y costo.

\section{Discusión}

El uso de nuevas tecnologías para realizar encuestas directas de opinión sustituyendo el papel y lápiz es una realidad que puede cambiar la forma de realizarlas, en esta investigación se evaluó la utilización de tabletas para que el tiempo en la encuesta fuera sensiblemente más rápido y así no tuviera pérdida de tiempo el entrevistado, ya que la investigación se realizo en un ciclo de conferencias y los entrevistados muchas veces no quieren realizarlas por el tiempo que implica sentarse y escribir, se utilizo Ipad 2 para la realización y se redujo el tiempo debido a la facilidad de realizar todo preestablecido.

De los seis tipos de coronas que se evaluaron en este estudio, el grupo 4 de coronas de fundas de celuloide (Strip Crown 3M ${ }^{\circledR}$ ), fue el mejor evaluado por los miembros de la AMOP. Esta situación concuerda con otros estudios previos que obtuvieron resultados similares, donde este tipo de coronas fueron la primera opción terapéutica para la restauración completa de incisivos afectados por caries o por trauma; ${ }^{14}$. Aunque no fue motivo de evaluación, los encuestados mencionaron que el problema con el uso de las coronas de fundas de celuloide, es lo sensible de la técnica al colocarlas, es decir, se debe considerar el control de hemorragia y saliva para evitar fracasos en la adhesión. ${ }^{15}$ Hubo diferencias significativas con los cinco grupos restantes, sobre todo con respecto a su bajo costo, pero sin tomar en cuenta la dificultad de la colocación (Tabla 4).

Las coronas de zirconia NuSmile ZR ${ }^{\circledR}$, (ZNS: grupo 2), fueron las segundas mejor evaluadas, observando que el color era lo más significativo a obtener; sin embargo, estudios de coronas de zirconia en dientes permanentes, muestran un color más estable, ademas de una estética superior debido a la baja porosidad. ${ }^{16,17}$ En relación a los dientes primarios existen pocos estudios sobre el color de la zirconia, sin embargo aunque varía de acuerdo a los diferentes tonos, no muestra cambios si no se contamina la zirconia. La recomendación de la casa comercial NuSmile es adquirir las coronas Try In (coronas de prueba color rosa) y así evitar problemas con el color de la zirconia en dientes primarios; sobre el uso de coronas de zirconia, se han reportado solo casos clínicos de su uso. Las coronas de zirconia tienen la ventaja de tener una fortaleza extraordinaria, y de soportar fuerzas masticatorias y de impacto oclusal sin fracturarse, ${ }_{1}^{18,19}$ sin embargo, no existen estudios que evalúen el desgaste de los dientes antagonistas a la corona de zirconia para dientes primarios; El grupo 1 tuvo una evaluación inferior a las coronas metal prefabricadas (NuSmile Signature ${ }^{\circledR}$, Grupo $5)$, es decir, que los entrevistados no encontraron diferencias entre la corona de zirconia EZ-Pedo y de metal prefabricada MES (6.41 vs 6.91). Un aspecto importante de mencionar es que los grupos 1, 2 y 5 , no tuvieron diferencias significativas en cuanto a ser la mejor elección para su uso en el consultorio dental, debido a que se consideraba el mismo precio para las tres opciones.

Las coronas metálicas fenestradas (grupo 6) obtuvieron una calificación de 4.6 colocándolas en la penúltima opción estética de preferencia por parte de los entrevistados; empero, estudios previos demuestran que es la opción estética más durable y su facilidad de reparación tiene una ventaja sobre 
las otras alternativas estéticas, pero se cuestiona su dificultad para implantarlas en dos sesiones, además que se tiene el inconveniente de observar el metal en el contorno de la ventana. ${ }^{19,20}$
La última opción fueron las coronas metálicas hechas en el consultorio (grupo 3), que aunque fueron las peor evaluadas, el conocimiento sobre su uso fue escaso; y lo criticable en su evaluación era

\begin{tabular}{|c|c|c|c|c|c|c|}
\hline \multirow[t]{2}{*}{ V. Ind } & \multirow[t]{2}{*}{ V. Dep } & \multirow[b]{2}{*}{ Prueba $t$} & \multirow[b]{2}{*}{$\begin{array}{c}\text { Error } \\
\text { estándar }\end{array}$} & \multirow[b]{2}{*}{ Sig. } & \multicolumn{2}{|c|}{$95 \%$ de intervalo de confianza } \\
\hline & & & & & $\begin{array}{l}\text { Límite } \\
\text { inferior }\end{array}$ & $\begin{array}{l}\text { Límite } \\
\text { superior }\end{array}$ \\
\hline \multirow[t]{5}{*}{1} & 2 & $-1.274^{*}$ & .254 & .000 & -2.00 & -.55 \\
\hline & 3 & $1.749^{*}$ & .254 & .000 & 1.02 & 2.48 \\
\hline & 4 & $-2.413^{*}$ & .256 & .000 & -3.14 & -1.68 \\
\hline & 5 & -.529 & .254 & .298 & -1.26 & .20 \\
\hline & 6 & $1.326^{*}$ & .254 & .000 & .60 & 2.05 \\
\hline \multirow[t]{5}{*}{2} & 1 & $1.274^{*}$ & .254 & .000 & .55 & 2.00 \\
\hline & 3 & $3.022^{*}$ & .253 & .000 & 2.30 & 3.75 \\
\hline & 4 & $-1.140^{*}$ & .255 & .000 & -1.87 & -.41 \\
\hline & 5 & $.744^{*}$ & .253 & .040 & .02 & 1.47 \\
\hline & 6 & $2.600^{*}$ & .253 & .000 & 1.88 & 3.32 \\
\hline \multirow[t]{5}{*}{3} & 1 & $-1.749^{*}$ & .254 & .000 & -2.48 & -1.02 \\
\hline & 2 & $-3.022^{*}$ & .253 & .000 & -3.75 & -2.30 \\
\hline & 4 & $-4.162^{*}$ & .255 & .000 & -4.89 & -3.43 \\
\hline & 5 & $-2.278^{*}$ & .253 & .000 & -3.00 & -1.55 \\
\hline & 6 & -.422 & .253 & .555 & -1.15 & .30 \\
\hline \multirow[t]{5}{*}{4} & 1 & $2.413^{*}$ & .256 & .000 & 1.68 & 3.14 \\
\hline & 2 & $1.140^{*}$ & .255 & .000 & .41 & 1.87 \\
\hline & 3 & $4.162^{*}$ & .255 & .000 & 3.43 & 4.89 \\
\hline & 5 & $1.884^{*}$ & .255 & .000 & 1.16 & 2.61 \\
\hline & 6 & $3.740^{*}$ & .255 & .000 & 3.01 & 4.47 \\
\hline \multirow[t]{5}{*}{5} & 1 & .529 & .254 & .298 & -.20 & 1.26 \\
\hline & 2 & $-.744^{*}$ & .253 & .040 & -1.47 & -.02 \\
\hline & 3 & $2.278^{*}$ & .253 & .000 & 1.55 & 3.00 \\
\hline & 4 & $-1.884^{*}$ & .255 & .000 & -2.61 & -1.16 \\
\hline & 6 & $1.856^{*}$ & .253 & .000 & 1.13 & 2.58 \\
\hline \multirow[t]{5}{*}{6} & 1 & $-1.326^{*}$ & .254 & .000 & -2.05 & -.60 \\
\hline & 2 & $-2.600^{*}$ & .253 & .000 & -3.32 & -1.88 \\
\hline & 3 & .422 & .253 & .555 & -.30 & 1.15 \\
\hline & 4 & $-3.740^{*}$ & .255 & .000 & -4.47 & -3.01 \\
\hline & 5 & $-1.856^{*}$ & .253 & .000 & -2.58 & -1.13 \\
\hline
\end{tabular}

Tabla 4. Comparaciones múltiples de las evaluaciones mediante la prueba HSD de Tukey 
la apariencia estética en relación con su color. Sin embargo, esto es contradictorio ya que la ventaja que tienen este tipo de coronas, es que se pueden utilizar diferentes tonos para tratar de mimetizar la corona con el diente adyacente, y además con la utilización de nuevos opacadores se elimina la desventaja de que se trasluzca el metal en el borde de las restauraciones. Cuando a algunos miembros encuestados que calificaban con menor puntaje al grupo 3, se les preguntó sobre qué alternativa estética usaban en su consulta, respondieron que ninguna, que seguían usando las coronas metálicas como primera opción.

Las preferencias de las coronas para su uso en el consultorio tuvo una clara preferencia hacia las coronas del grupo 4, siendo la única y primera opción por parte de los estudiantes. Lo que comprueba los resultados reportados por Bradley, donde reporta que en el $84 \%$ de los posgrados no enseñaban el uso de coronas de zirconia. ${ }^{22}$

Es importante destacar que este estudio es la primera investigación realizada a odontólogos pedia- tras miembros de la AMOP, ya que los estudios de satisfacción y preferencias relacionados con la estética, se han realizado solamente a padres o tutores de pacientes. ${ }^{21}$

\section{Conclusiones}

Las encuestas realizadas con medios tecnológicos (tabletas de respuesta rápida) muestran una disposición mayor de los entrevistados a realizar encuestas de investigación.

Las coronas de funda de celuloide son la alternativa preferida por parte de los odontopediatras encuestados miembros de la AMOP. Empero, las coronas de zirconia son una alternativa nueva y con buena aceptación. Asimismo, no existen diferencias significativas entre las coronas metálicas con frente estético y las coronas de zirconia. Es recomendable que las Escuelas de Odontopediatría tengan como opción el entrenamiento para el uso de todas las alternativas mencionadas, para que el estudiante de posgrado tenga un amplio criterio en el uso de las diferentes alternativas estéticas.

\section{Referencias bibliográficas}

1.Gosnell ES, Thikkurissy S. Management of dental caries and esthetic is-sues in the pediatric patient. J Calif Dent Assoc 2013;41(8):619-29.

2. American Academy of Pediatric Dentistry; American Academy of Pediatrics. Early childhood caries (ECC): Classifications, Consequences, and Preven-tive Strategies. Pediatr Dent 2014;35(6):50-2.

3. Elqadir AJ, Shapira J, Ziskind K, Ram D. Esthetic restorations of primary an-terior teeth. Refuat Vehashinayim 2013;30(2):54-60

4. Berg JH. The continuum of restorative materials in pediatric dentistry- a re-view for the clinician. Pediatr Dent 1998;20(2): 93-100.

5. Lee JK. Restoration of primary anterior teeth: review of the literature. Pediatr Dent. 2002;24(5):506-10

6. Waggoner WF. Restoring primary anterior teeth. Pediatr Dent 2002;24(5):511-6.

7. Kopel HM, Beaver HA. Comprehensive restorative procedures for primary anteriors. J Dent Child 1967;34(5):412-23.

8. Webber DL, Epstein NB, Wong JW, Tsamtsouris A. A method of restoring primary anterior teeth with the aid of a celluloid crown form and composite resins. Pediatr Dent 1979;1(4):244-6.

9. Croll TP, Helpin ML. Preformed resin-veneered stainless Steel crowns for restoration of primary incisors. Quintessence 1996;27(5):309-13. 
10. Croll TP. Primary incisor restoration using resin-veneered stainless steel crowns. ASDC J Dent Child. 1998;65(2):89-95.

11. Guelmann M, Gehring DF, Turner C. Retention of veneered stainless steel crowns on replicated typodont primary incisors: an in vitro study. Pediatr Dent 2003;25(3):275-8.

12. Karaca B, Ozbay G, Kargul B. Primary zirconia Crown restorations for chil-dren with early childhood caries. Acta Stomatol Croata. 2013;47(1):64-71.

13. Planells del Pozo P, Fuks AB. Zirconia crowns - an esthetic and resistant restorative alternative for ECC affected primary teeth. J Clin Pediatr Dent 2014;38(3): 193-5.

14. Saha R, Malik P. Paediatric aesthetic dentistry: a review. Eur J Paediatr Dent 2012;13(1):6-12.

15. Croll TP, Berg J. Simplified primary incisor proximal restoration. Pediatr Dent 2003;25(1):67-70.

16. Manicone PF, Rossi Iommetti P, Raffaelli L. An overview of zirconia ceram-ics: Basic properties and clinical applications. J Dent 2007;35(11):819-26.

17. Larsson C. Zirconium dioxide based dental restorations. Studies on clinical performance and fracture behaviour. Swed Dent J Suppl 2011;(213):9-84.

18. Croll TP, Donly KJ. Contralateral/opposite-arch zirconia crown, restoration of two mandibular primary first molars: A tooth-colored solution to restoring damaged primary teeth. Inside Dent 2015;11(3): 78-81.

19. Croll TP, Jefferies SR, Writght JT. Primary Incisor and Canine Restoration in a Child with Amelogenesis Imperfecta: Material advances bring new op-tions to the treatment of enamel malformations. Inside Dent 2014;10(12):1-4

20. Yilmaz Y, Koçoğullari ME. Clinical evaluation of two different methods of stainless steel esthetic crowns. J Dent Child (Chic) $2004 ; 71(3): 212-4$.

21. Champagne C, Waggoner W, Ditmyer M, Casamassimo PS, MacLean J. Parental satisfaction with preveneered stainless steel crowns for primary anterior teeth. Pediatr Dent 2007;29(6):465-9.

22. Bradley K, Owens D, Harvey D, Oueis H. Use of Zirconia Crowns by Pedi-atric Dentists. Childrens Hospital of Michigan, American Academy of Pedi-atric Dentistry, Seattle W. Poster 265. 2015.

Recibido: $15 / 08 / 2016$

Aceptado: 10/11/2016

Correspondencia: Héctor A. Ramírez Peña, DDS.

Paseo de los Leones \#145-4 (Plaza Cristal) Col. Cumbres Elite, Monterrey NL,

E-mail: odontologiapediatrica@gmail.com 